\title{
Repercussões cardiovasculares do uso de inibidores de SGLT2 em portadores de Diabetes Mellitus tipo 2
}

\author{
Cardiovascular repercussions of the use of SGLT2 inhibitors in patients with type 2 \\ Diabetes Mellitus
}

\section{Repercusiones cardiovasculares del uso de inhibidores de SGLT2 en pacientes con Diabetes Mellitus tipo 2}

Thaiz Geovana Bezerra1*, Ana Luiza Gomes Gonçalves², Andrezza Mendes Franco ${ }^{3}$, Bruna Araújo Rossi $^{4}$, Gabriela Aparecida Schiefler Gazzoni5, Marcella Lourenço Winter ${ }^{6}$, Mariana Oshiro Marques ${ }^{7}$, Sophia Bark ${ }^{8}$, Vinícius Sippel Cruz $^{9}$, Rachel Cardoso Lopes Rêgo ${ }^{6}$.

\section{RESUMO}

Objetivo: Avaliar os efeitos cardiovasculares dos inibidores do Transportador 2 de Sódio-Glicose (SGLT2i) e estabelecer seus benefícios em pacientes portadores de Diabetes Mellitus (DM). Revisão Bibliográfica: Os pacientes que apresentam DM possuem maior predisposição à eventos cardiovasculares, tendo em vista 0 estresse oxidativo que ocorre devido às alterações metabólicas inerentes à patologia. Os fármacos inibidores da SGLT2 possuem atuação renal ao impedir que os transportadores SGLT2 realizem a reabsorção tubular da glicose. Estudos revelam benefícios clínicos de seu uso em pacientes diabéticos, medidos através da diminuição das internações por insuficiência cardíaca e da ocorrência de infarto agudo do miocárdio quando comparado com outras terapias hipoglicemiantes. Além disso, eles auxiliam na prevenção de distúrbios renais, como o dano glomerular por hiperglicemia e na perda de massa corporal. Considerações finais: Os inibidores do SGLT2 são fármacos eficazes no tratamento do DM2 e possuem contribuição importante na prevenção e/ou controle de doenças cardiovasculares e renais e suas repercussões clínicas.

Palavras-chave: Inibidores do transportador 2 de sódio-glicose, Sistema cardiovascular, Diabetes mellitus.

\begin{abstract}
Objective: To evaluate the cardiovascular effects of Sodium-Glucose Transporter 2 inhibitors (SGLT2i) and establish their benefits in relation to patients with Diabetes Mellitus (DM). Bibliographic Review: Patients with DM have a greater predisposition to cardiovascular events due to the oxidative stress in this sistem from the metabolic alterations present in this pathology. SGLT2 inhibitory drugs present renal action by preventing SGLT2 transporters in the kidney from reabsorbing tubular glucose. Studies reveal clinical benefits of its use in diabetic patients, measured by decreasing hospitalizations for heart failure and the occurrence of disorders like heart attacks, when compared with other hypoglycemic therapies. In addition, they help prevent kidney disorders, such as glomerular damage caused by hyperglycemia, and reduce body mass, another aspect that contributes to the prevention of circulatory disorders. On the other hand, they have adverse effects, which should be taken into account when opting for a therapy using SGLT2i-based drugs on patients with type 2 Diabetes Mellitus. Final considerations: SGLT2 inhibitors are effective drugs in the treatment of DM2 and have an important contribution in the prevention and / or control of cardiovascular and renal diseases and their clinical repercussions.
\end{abstract}

Key words: Sodium-glucose transporter 2 inhibitors, Cardiovascular system, Diabetes mellitus.

\footnotetext{
${ }^{1}$ Universidade do Oeste Paulista (UNOESTE), Jaú - SP. * E-mail: thaizgeovanabezerra@gmail.com

2 Universidade de Cuiabá (UNIC), Cuiabá - MT.

${ }^{3}$ Universidade Federal do Amazonas (UFAM), Manaus - AM.

${ }^{4}$ Universidade federal do Rio de Janeiro (UFRJ), Macae - RJ.

5 Universidade do Vale do Itajaí (UNIVALI), Itajaí - SC.

${ }^{6}$ Faculdade Ciências Médicas de Minas Gerais (FCMMG), Belo Horizonte - MG.

7 Universidade Federal de Mato Grosso (UFMT), Cuiabá - MT.

8 Instituto Presidente Antônio Carlos (ITPAC), Palmas - TO.

${ }^{9}$ Faculdade Evangélica Mackenzie do Paraná (FEMPAR), Curitiba - PR.
} 


\section{RESUMEN}

Objetivo: Evaluar los efectos cardiovasculares de los inhibidores del Transportador de Sodio-Glucosa 2 (SGLT2i) y establecer sus beneficios en pacientes con Diabetes Mellitus (DM). Revisión bibliográfica: Los pacientes con DM tienen una mayor predisposición a los eventos cardiovasculares, ante el estrés oxidativo que se produce por los cambios metabólicos inherentes a la patología. Los fármacos inhibidores de SGLT2 tienen acción renal al evitar que los transportadores de SGLT2 realicen la reabsorción tubular de glucosa. Los estudios revelan los beneficios clínicos de su uso en pacientes diabéticos, medidos por la disminución de las hospitalizaciones por insuficiencia cardíaca y la aparición de infarto agudo de miocardio en comparación con otras terapias hipoglucémicas. Además, ayudan a prevenir trastornos renales, como el daño glomerular por hiperglucemia y pérdida de masa corporal. Por otro lado, tienen efectos adversos que deben tenerse en cuenta a la hora de optar por utilizar este método de control glucémico en pacientes con Diabetes Mellitus tipo 2. Consideraciones finales: Los inhibidores de SGLT2 son fármacos eficaces en el tratamiento de la DM2 y tienen una importante contribución en la prevención y / o control de las enfermedades cardiovasculares y renales y sus repercusiones clínicas

Palabras clave: Inhibitores del cotransportador de sodio-glucosa 2, Sistema cardiovascular, Diabetes mellitus.

\section{INTRODUÇÃO}

O Diabetes Mellitus (DM) caracteriza-se por ser uma doença crônica, relacionada à incapacidade do organismo de sintetizar o hormônio insulina de forma suficiente ou de empregá-la corretamente para controle dos níveis plasmáticos de glicose (KAPLAN A, et al., 2018). Devido à fisiopatologia do DM e sua correlação com a obesidade, hipertensão e dislipidemia, os riscos de desenvolvimento de doenças cardiovasculares associadas são significativos, visto que, é causa de doença renal em estágio terminal e está associado a um risco elevado de insuficiência cardíaca (LAYTON AT e VALLON V, 2018). Além disso, os pacientes com DM apresentam até quatro vezes mais chance de desenvolver doença arterial coronariana e infarto do miocárdio, estabelecendo que DM se faz um fator de risco independente para acidente vascular cerebral e doenças cardíacas (DE ROSA S, et al., 2018).

Atualmente, o DM é um problema de saúde global e cerca de $8 \%$ da população mundial apresenta esta patologia. Sabe-se que a estimativa do número de acometidos com a doença pode chegar a 592 milhões nos próximos 25 anos, sendo que a América do Sul e a América Central contribuem com 24 milhões de doentes (MATTA-HERRERA GJ, et al., 2018).

Durante décadas, as principais formas de tratamento do DM incluíram sulfoniluréias e inibidores de DPP4, dentre os quais o único alvo terapêutico era o controle glicêmico e não a redução de eventos cardiovasculares. Com o passar dos anos e devido à grande importância, o risco cardiovascular dos pacientes com DM foi levado em consideração para o desenvolvimento de novos medicamentos. Desta forma, os inibidores do co-transportador 2 de sódio-glicose (SGLT2i) são considerados a primeira classe de medicamentos para a terapia do DM que demonstrou redução do risco cardiovascular (MARTINS EB, et al., 2020).

Os SGLT2 inibem a reabsorção de glicose e sódio no túbulo contorcido proximal renal, têm ganhado destaque no tratamento da DM, ao reduzirem a glicemia de uma forma insulino-dependente e contribuírem para que haja uma melhora da função das células beta pancreáticas e da sensibilidade à insulina (CINTRA R, et al., 2019). Representados pela empagliflozina, dapagliflozina e canagliflozina, os inibidores de SGLT2i mostraram resultados positivos em relação a doenças cardiovasculares (DCV) ao contribuir para a redução da mortalidade, internações por insuficiência cardíaca, redução da albuminúria e manutenção, em longo prazo, da taxa de filtração glomerular (GONZALEZ DE, et al., 2020).

Ainda, os inibidores de SGLT2i mostraram diminuir o estresse da parede cardíaca, reduzindo a progressão da insuficiência cardíaca (VERMA S e MCMURRAY JJV, 2018). Consequentemente à inibição do SGLT2, há o aumento da glicosúria, o que auxilia na perda de peso. Dessa maneira, o impacto na hemodinâmica e transporte tubular garantidos pelos inibidores de SGLT2i mostraram ser efetivos e encontram-se nas 
vantagens clinicamente significativas em termos de proteção de pacientes diabéticos contra insuficiência cardíaca e renal (LAYTON AT e VALLIN V, 2018).

De forma geral, os inibidores do SGLT2 possuem um bom perfil de segurança, sendo bem tolerados e seguros (GONZALEZ DE, et al., 2020). Em consequência da natriurese e do efeito diurético osmótico, pode haver diminuição da pressão arterial sistólica e, em consequência, hipotensão arterial (GARCIA-ARIAS MR, et al., 2018).

Dada a elevada prevalência e incidência do diabetes mellitus no mundo e considerando as diversas complicações micro e macrovasculares associadas, objetivou-se destacar a classe dos inibidores do SGLT2 como um dos principais fármacos anti-diabéticos, a qual visa minimizar os impactos da diabetes e suas complicações associadas. Sob essa perspectiva, o presente artigo busca, através de revisão de literatura, que essas drogas despontam como grandes aliadas no manejo terapêutico para os portadores de diabetes, principalmente no que se refere aos benefícios cardiovasculares. Portanto, serão descritos alguns dos principais mecanismos que podem explicar os notáveis benefícios cardioprotetores dos inibidores de SGLT2, incluindo eficácia efeitos no volume e diurese, metabolismo miocárdico e efeitos renais.

\section{REVISÃO BIBLIOGRÁFICA}

\section{Farmacologia dos Inibidores de SGLT2}

Os rins são os órgãos envolvidos no mecanismo de ação dos fármacos inibidores da SGLT2 (GARCíAARIAS MR, et al., 2018). Em condições fisiológicas, esse órgão apresenta grande importância no controle dos níveis glicêmicos, visto que são responsáveis pela filtração e reabsorção de glicose. Nesse processo, a SGLT2 atua como transportador no segmento inicial do túbulo proximal com o objetivo de realizar a reabsorção da maior parte da glicose excretada (MATTA-HERRERA GJ, et al., 2018). Com isso, os SGLT2i atuam de forma a inibir os transportadores SGLT2, favorecendo a excreção de glicose e controlando os níveis glicêmicos em pacientes que apresentam diabetes mellitus 2 (DM2) (GARCÍA-ARIAS MR, et al., 2018).

Além do controle glicêmico nesses pacientes, esses fármacos parecem estar associados a outros efeitos benéficos ao sistema cardiovascular, aos rins e à perda de peso, principalmente em idosos, porém, esses fármacos não estão isentos de efeitos colaterais o que tende analisar as condições fisiopatológicas durante a sua prescrição (CINTRA R, et al., 2019; SANTOS LL, et al., 2016). Os inibidores SGLT2 estão presentes nas formas terapêuticas dos medicamentos empagliflozina, canagliflozina e dapagliflozina (PEREL, 2018; MARTINS E, et al., 2020). Eles têm características farmacocinéticas e farmacodinâmicas semelhantes, exceto pelo grau de seletividade do bloqueio dos receptores SGLT-2 em relação aos receptores SGLT-1 (MARTINS E, et al., 2020).

\section{Principais efeitos cardiovasculares}

O risco de doença cardiovascular, em pacientes com DM2, é aumentado em até 4 vezes, pois cerca de $65 \%$ dos pacientes que desenvolveram quadro de infarto agudo do miocárdio possuem DM2 ou redução da tolerância à glicose, sendo que a presença desse tipo de diabetes um contribuinte para o risco de mortalidade e desenvolvimento de insuficiência cardíaca congestiva (KAPLAN A, et al., 2018). A justificativa para as doenças cardiovasculares serem responsáveis pela morte de pessoas acometidas por DM2 é devido a relação entre ambas as patologias que resulta em doenças que afetam a integridade das artérias, sendo representadas, portanto, pela doença arterial coronariana e doença arterial periférica que por sua vez pode contribuir para complicações severas e a possibilidade de mortalidade aumentada (DIEUZEIDE G, et al., 2018).

Dessa forma, a escolha terapêutica que inclui os medicamentos inibidores de SGLT2 aumentou de maneira significativa para os pacientes que apresentam o risco de desenvolver doenças cardiovasculares, pois quando presentes na composição de medicamentos são promissores para a proteção e combate de afecções relacionadas ao sistema cardiovascular (TENTOLOURIS A, et al., 2019; SRIDHAR VS, et al., 2020). Com isso, o uso combinado com medicamentos já utilizados em pacientes que possuem DM2 aumentou de 
maneira semelhante, principalmente em pacientes com múltiplas comorbidades além das metabólicas como forma de lhes proporcionar proteção e inibição de potenciais eventos cardiovasculares (NOLAN CJ, et al., 2011; SRIDHAR VS, et al., 2020). Novos estudos clínicos, com a participação de indivíduos não diabéticos que apresentam comprometimento de suas funções renais e cardiovasculares, confirmam os benefícios dos iSGLT2, o que comprova que sua capacidade de se tornar mais uma das opções terapêuticas para os profissionais prescritores (SRIDHAR VS, et al., 2020; ZINMAN B, et al., 2015).

Diversos estudos, como o EMPA-REG OUTCOME e o LEADER, foram realizados com o intuito de identificar uma relação fidedigna entre o uso de inibidores de SGLT2 e as repercussões cardiovasculares benéficas em pacientes com DM2, sendo os SGLT2i, a primeira classe de antidiabéticos comprovadamente eficaz nesse aspecto (PEREL C, 2018; ZINMAN B, et al., 2015; MARSO SP, et al., 2013). Um deles foi o estudo EMPAREG, que estudou uma população de pacientes que apresentam DM2 com doença cardiovascular estabelecida e muito alto risco cardiovascular, dividida em um grupo que recebia a Empagliflozina e outro que recebia placebo. Como resultados, foi verificada uma redução de cerca de $14 \%$ na ocorrência de eventos como Acidente Vascular Cerebral, Infarto Agudo do Miocárdio e Morte Cardiovascular, além de uma redução de $38 \%$ na prevalência de doenças cardiovasculares, $32 \%$ de todas as causas de morte e ainda de $35 \%$ em hospitalizações por doenças do aparelho circulatório (ZINMAN B, et al.,2015).

Outro estudo que se destacou nesse âmbito foi o CANVAS, que teve como população-alvo indivíduos com 30 anos ou mais e diagnóstico prévio de doenças cardiovasculares, além de um grupo de pessoas com 50 anos ou mais que tinham fatores de risco para o desenvolvimento de doenças cardiovasculares, configurandose como um grupo de prevenção primária. Os participantes do estudo foram divididos, assim como no anterior, em grupo caso e placebo, em que no grupo caso foi administrado o fármaco canagliflozina. Como desfechos do estudo, foi constatada uma diminuição geral nas taxas de morte cardiovascular, Acidente Vascular Cerebral e Infarto do Miocárdio não fatal, assim como redução das hospitalizações por insuficiência cardíaca. Todavia, quando é analisada somente a amostra de pacientes da prevenção primária, os resultados não demonstraram diminuições significativas de nenhum dos desfechos observados no grupo como um todo (NEAL B, et al., 2017)

Comparando os resultados desses dois estudos, nota-se que no EMPAREG as repercussões do uso da empagliflozina foram mais evidentes do que no CANVAS, com a canagliflozina. Isso pode desencadear a indagação de que a empagliflozina poderia ser mais eficiente que a canaglifozina no combate às complicações cardiovasculares do DM2, ou essa diferença poderia ser resultante das diferenças entre as populações avaliadas, uma vez que no CANVAS cerca de um terço dos participantes pertenciam ao grupo de prevenção primária (D'EMDEN M, et al., 2017; UTHMAN L, et al., 2018).

Em pacientes com insuficiência cardíaca, o uso de SGLT2i promoveu efeitos protetores relacionados com os mecanismos de pré-carga, pós-carga e contratilidade do coração. A pré-carga é reduzida ao inibir o transportador SGLT2. O mecanismo parece ocorrer por aumento da diurese osmótica, reduzindo o volume plasmático, o que diminui a sobrecarga ao miocárdio e melhora a distensão e contratilidade do coração. A redução da pós-carga em pacientes com DM2 tratados com a empagliflozina é decorrente da redução da resistência vascular e da pressão arterial (KAPLAN A, et al., 2018).

A disfunção cardíaca em pacientes com DM2 pode estar relacionada com a redução da eficiência de contração e relaxamento das câmaras cardíacas, aumento do risco de arritmias devido ao aumento da atividade simpática e alterações em relação a homeostase iônica e fisiologia cardíaca. O miocárdio desses pacientes também está exposto ao dano oxidativo e inflamação, que são decorrentes de alteração metabólica geradas pelo curso da doença metabólica (KAPLAN A, et al., 2018).

O SGLT2i parece ter ainda impacto positivo contra o dano oxidativo e inflamação cardíaca. A utilização da empagliflozina em ratos pré-diabéticos diminui a hipertrofia das células musculares cardíacas, fibrose e tecido adiposo subcutâneo. Dessa forma, houve redução do dano oxidativo e da inflamação, o que parece ser o efeito protetor cardíaco do SGLT2i. Além disso, foi realizada a administração do medicamento dapagliflozina em ratos, de forma a determinar o impacto da modulação da inflamação cardíaca desses fármacos. Os 
resultados mostraram uma grande regulação de citocinas anti-inflamatórias, o que sustentou o mecanismo dos SGLT2i como fármacos controladores do dano oxidativo e inflamatório cardíaco (KAPLAN A, et al., 2018).

Os inibidores de SGLT2, quando comparados com as demais terapêuticas hipoglicemiantes que utilizam insulina exógena ou medicações que estimulam a secreção de insulina endógena, apresentaram menores taxas de mortalidade por insuficiência cardíaca em diabéticos com doenças cardiovasculares preexistentes. Tais medicações, através da elevação dos níveis de insulina, aumentam a captação de substratos energéticos como ácidos graxos e glicose pelo coração, promovendo elevação na gênese de excretas lipídicas tóxicas, como diacilglicerol e ceramidas, e aumento do mecanismo de glicação, processos altamente lesivos ao tecido cardíaco (SILVA G, et al., 2020).

O transporte de glicose e de sódio ( $\mathrm{Na}+$ ) são acoplados, o que possibilita que a inibição de SGLT2 provoque uma reabsorção de $\mathrm{Na}+$ túbulo proximal, o que induz um efeito na pressão arterial. A diminuição significativa está compreendida para a pressão arterial sistólica entre 3 a $5 \mathrm{mmHg}$ e de 1 a $3 \mathrm{mmHg}$ para a pressão arterial diastólica (LAYTON AT e VALLON V, 2018). O infarto agudo do miocárdio é uma das complicações relacionadas com pacientes acometidos por DM2, o qual os resultados se têm demonstrado positivos após tratamento com inibidor de SGLT2, principalmente, quando os pacientes são submetidos a monoterapia. Contudo, as doenças cerebrovasculares, que têm o acidente vascular cerebral isquêmico como principal agravante, não apresentam diferença na incidência após tratamento com inibidores de SGLT2 em pacientes com DM2 mesmo com a monoterapia (LUCONI M, et al., 2016).

\section{Efeitos renais dos fármacos SGLT2i}

O DM é um dos principais fatores de risco para o desenvolvimento da doença renal (DR) (CINTRA R, et al., 2019). Isso ocorre devido a mudanças funcionais decorrentes do estado hiperglicêmico e mecanismos fisiopatológicos que resultam em lesão dos glomérulos. O uso de SGLT2i promove benefícios hemodinâmicos, além da redução do nível glicêmico, ambos importantes no tratamento da DR. A inibição do SGLT2 proporciona efeito nefroprotetor pois a redução do volume circulante aumenta a atividade dos bloqueadores do sistema renina-angiotensina-aldosterona circulantes que são responsáveis pela proteção da unidade funcional dos rins. No entanto, a administração dos SGLT2i deve ser evitada em pacientes que apresentam insuficiência renal com taxa glomerular inferior a $45 \mathrm{mil} / \mathrm{min}$, visto que a ação do medicamento depende da filtração renal (MORALES-OLVERA D, et al.,2020).

Além disso, ao inibir os transportadores SGLT2 há redução da absorção da glicose, o que aumenta o nível de sódio na mácula densa (porção distal dos túbulos renais), resultando em vasoconstrição aferente, reduzindo tanto a filtração glomerular, quanto a pressão dentro do glomérulo, que são mecanismos fisiopatológicos da DR (GONZALEZ DE, et al., 2020). Um outro trabalho dividiu os mecanismos de proteção renal, decorrente do uso de SGLT2i, em mecanismos diretos e indiretos. Os mecanismos indiretos foram aqueles que geraram o controle glicêmico, redução do peso e redução da pressão arterial. Já os mecanismos diretos compreendem aqueles que resultaram na pressão intraglomerular reduzida, com redução da filtração glomerular por vasoconstrição da artéria aferente, e atenuação de fatores inflamatórios nos rins e diminuição da isquemia renal, restringindo a lesão do rim (SRIDHAR VS, et al., 2020). Logo, os fármacos inibidores SGLT2 são vistos como uma grande ferramenta contra doenças renais (GONZALEZ DE, et al., 2020).

O retardo na progressão da nefropatia diabética é evidente na utilização da empagliflozina em modelo in vivo ao demonstrar que nas células do túbulos contorcidos proximal ocorre a diminuição do efeito próinflamatório dessa estrutura devido ao bloqueio da entrada de glicose decorrente da falta do processo de reabsorção. (MORALES-OLVERA D, et al., 2017) Contudo, cabe ressaltar que efeitos anti-hiperglicêmicos dos inibidores de SGLT2 são atenuados em pacientes com taxa de filtração glomerular reduzida, o que implica nos seus benefícios supracitados (LAYTON AT, VALLON V, 2018).

\section{Redução na massa corporal}

O uso SGLT2i está relacionado à perda de peso induzida, mesmo em pacientes que fazem uso de outras medicações que podem ter como efeito colateral o ganho de peso. O mecanismo de perda de peso ocorre por meio da glicosúria causada pelos fármacos SGLT2i. Além disso, a perda de peso induzida não está 
relacionada ao aumento da mortalidade em idosos, visto que a redução da massa corporal em maior parte se dá pela perda de gordura, o que parece melhorar a função física (CINTRA R, et al, 2019). Outro aspecto importante é que a redução de peso promove atenuação do risco cardiovascular e eleva a sensibilidade à insulina (HENNING RJ, et al., 2018).

As gliflozinas demonstram ser eficazes em relação a perda de peso, ocasionando uma redução média de $2,1 \mathrm{~kg}$ quando comparado com o grupo placebo após uso por um período de 12 semanas e uma redução média de 2,9 kg para um período de uso superior a dois anos. Tal efeito positivo para a redução da massa corporal se deve ao déficit calórico de aproximadamente $50 \mathrm{kcal} /$ dia que proporciona também a redução dos níveis plasmáticos de insulina. Com isso, se tem redução de peso decorrente da perda de tecido adiposo relacionado aos órgãos e ativação do tecido adiposo marrom (LIMA JUNIOR JC, et al., 2019). Considera-se, portanto, que o uso do SGLT2i associado à dieta e exercícios irá proporcionar a redução de peso, mas o uso desse fármaco não é a primeira escolha para tratamento da obesidade (HENNING RJ, et al., 2018).

\section{Efeitos Adversos dos fármacos SGLT2i}

Na prática clínica, a escolha do medicamento para o tratamento de DM2 deve ser realizada considerando a sua eficácia e segurança, efeitos colaterais, contraindicações, tolerabilidade, adesão terapêutica e adequação para o paciente a fim de proporcionar o melhor tratamento. Dessa forma, é importante se basear no perfil de risco do paciente, ou seja, a escolha de ser individualizada, com o objetivo de atingir níveis glicêmicos ideais. (DIEUZEIDE G, et al., 2018). Apesar dos benefícios cardiovasculares e renais observados com o uso de empagliflozina, canagliflozina e dapagliflozina, parecem existir diferenças importantes em relação aos seus perfis de segurança. Os efeitos adversos mais comuns com SGLT2i são explicados pelo mecanismo de ação renal específico dessa classe farmacológica. Infecções genitais micóticas ocorreram 4 a 6 vezes mais com SGLT2i comparados ao placebo ou outros agentes antidiabéticos. Apesar de aumentar o risco de infecções genitais, seu uso não altera o de infecções do trato urinário. (D'EMDEN M, et al., 2017; SCHEEN J, et al., 2018; TOYAMA T, et al., 2019; ZELNIKER T, et al., 2019).

Ademais, a terapia com inibidores de SGLT2 aumenta o efeito natriurético dos diuréticos tiazídicos e de alça como a furosemida e, portanto, pode aumentar o risco de desidratação e hipotensão. Mas são eventos considerados raros e não severos. No entanto, como pontos práticos em torno da segurança ao se considerar o uso do SGLTi, é prudente sempre verificar o risco de infecções geniturinárias, além de observar a depleção de volume na existência de doença concomitante ou clima quente podendo causar desidratação, ou, ainda, quando estão em uso de diuréticos de alça (D'EMDEN M, et al., 2017; SCHEEN J, et al., 2018).

Os inibidores SGLT2 também podem aumentar o risco de cetoacidose normoglicêmica, um efeito adverso raro, mas potencialmente fatal para essa classe de medicamentos. A redução na dose de insulina, a estimulação da liberação de glucagon e a reabsorção de cetona aumentada nos túbulos renais podem contribuir para o aumento na concentração de corpos cetônicos, ao passo que a glicosúria intensificada limita a amplitude da hiperglicemia. (SCHEEN J, et al., 2018; TOYAMA T, et al., 2019; USMAN MS, et al., 2018). O risco pode ser reduzido com orientação adequada ao paciente e vigilância (ZELNIKER T, et al., 2019).

Outra complicação grave é a amputação periférica. (SCHEEN J, et al., 2018) O uso de canagliflozina, como demonstrado no Programa CANVAS, aumentou o risco de amputações e fraturas ósseas (respectivamente, 6,3 versus 3,4 participantes por 1000 pacientes-ano; HR, 1,97; IC 95\%, 1,41 -2,75). (D'EMDEN M, et al., 2017; SCHEEN J, et al., 2018; TOYAMA T, et al., 2019; SCHEEN J, et al., 2018). Entretanto, nenhum risco aumentado de amputações foi relatado no estudo EMPA-REG OUTCOME, com empagliflozina versus placebo (HR, 1,00; IC de 95\%, 0,70-1,44) (SCHEEN J, et al., 2018). Atualmente, pouco se sabe sobre os efeitos adversos dessa classe de medicamento em pacientes não diabéticos, estudos de larga escala são necessários para maiores investigações. (USMAN MS, et al., 2018).

\section{CONSIDERAÇÕES FINAIS}

É possível constatar que os inibidores do SGLT2 são fármacos eficazes no tratamento do DM2 e possuem contribuição importante na prevenção e/ou controle de doenças cardiovasculares e renais e suas repercussões clínicas. Por atuar a nível renal provocando glicosúria, tem como efeitos globais a redução dos 
danos oxidativos e inflamatórios cardíacos, melhora da distensão e contratilidade miocárdica, redução da sobrecarga volumétrica na pré e pós-carga, diminuição das lesões renais e aumento da perda de peso induzida. Por conta disso, o uso desses fármacos impacta positivamente na redução de tempo de hospitalização e taxas de óbitos por IAM em indivíduos diabéticos. Ademais, um bom perfil de segurança e poucos efeitos adversos são motivos pelos quais essa classe de medicamentos merece notoriedade no âmbito das condutas terapêuticas nesse grupo de pacientes. Todavia, é importante ressaltar que os efeitos benéficos advindos do uso dessas medicações não excluem o impacto negativo dos demais fatores de risco cardiovasculares.

\section{REFERÊNCIAS}

1. CINTRA R, et al. Inibição do co-transportador 2 de sódio-glicose em idosos: percepções clínicas e mecanísticas sobre segurança e eficácia. Rev. Assoc. Med. Bras., 2019; 65(1): 70-86.

2. DE ROSA S, et al. Type 2 diabetes mellitus and cardiovascular disease: genetic and epigenetic links. Frontiers in endocrinology, 2018; 9: 1-13.

3. D'EMDEN M, et al. SGLT2 inhibitors with cardiovascular benefits: transforming clinical care in Type 2 diabetes mellitus. Diabetes Research and Clinical Practice, 2017; 136: 23-31.

4. DIEUZEIDE G, et al. Efectos cardiovasculares de los nuevos fármacos no insulínicos en diabetes. Revista Medicina, 2018; 78(3): 185-193.

5. GARCIA-ARIAS MR, et al. Efeito cardiometabólico dos inibidores do cotransportador de glicose de sódio tipo 2 (SGLT2). Med. Interna Mex, 2018; 34(6): 924-932.

6. GONZALEZ DE, et al. Inibidores do SGLT-2 no diabetes: um enfoque na renoproteção. Rev. Assoc. Med. Brasil., 2020; 66(1): 17-24.

7. HENNING RJ. Type-2 diabetes mellitus and cardiovascular disease. Future Cardiol, 2018; 14(6): 491-509.

8. KAPLAN A, et al. Direct cardiovascular impact of SGLT2 inhibitors: mechanisms and effects. Heart Failure Reviews, 2018; 23(3): 419-437.

9. LAYTON AT, VALLON V. Cardiovascular benefits of SGLT2 inhibition in diabetes and chronic kidney diseases. Acta physiologica, 2018; 222(4): e13050.

10. LIMA JUNIOR JC, et al. Papel central da obesidade na disfunção das células endoteliais e risco cardiovascular. Rev. Assoc. Med. Bras, 2019; 65 (1): 87-97.

11. LUCONI M, et al. Which is the main molecular target responsible for the cardiovascular benefits in the EMPA-REG OUTCOME trial? A journey through the kidney, the heart and other interesting places. Nutrition, Metabolism and Cardiovascular Diseases, 2016; 26(12): 1071-1078.

12. MARSO SP, Poulter NR, Nissen SE et al. Design of the liraglutide effect and action in diabetes: evaluation of cardiovascular outcome results (LEADER) trial. Am Heart J., 2013;166:823-830.

13. MARTINS EB, et al. Pharmacological therapy and cardiovascular risk reduction for type 2 diabetes. Revista associação médica brasileira, 2020; 66(9): 1283-1288

14. MATTA-HERRERA GJ, et al. Agonistas de GLP-1 mais inibidores de SGLT2. Efeitos cardioprotetores aditivos?. Med. Interna Mex, 2018; 34(4): 601-613;

15. MORALES-OLVERA, D et al. iSGLT2 y su potencial efecto nefroprotector en pacientes con diabetes mellitus 2 . Med. interna Méx., 2017; 33(4): 503-510.

16. NEAL B, et al. Canagliflozin and Cardiovascular and Renal Events in Type 2 Diabetes. New England Journal of Medicine, 2017; 377(7), 644-657.

17. NOLAN CJ, et al. Type 2 diabetes across generations: from pathophysiology to prevention and management. The Lancet, 2011; 378(9786): 169-181.

18. PEREL C. Insuficiencia cardíaca y diabetes: Nuevos tratamientos para la diabetes. Insuf Card, 2018; 13(4): 155-169.

19. SANTOS LL, et al. Uso de inibidores SGLT-2 no tratamento do diabetes mellitus tipo 2. Rev. Assoc. Med. Bras., 2017; 63(7): 636-641.

20. SCHEEN AJ, et. al. Cardiovascular effects of new oral glucose-lowering agents: DPP-4 and SGLT-2 inhibitors. Circulation research, 2018; 122(10): 1439-1459.

21. SILVA G, et al. Inibidores da SGLT2 e suas influências no sistema cardiovascular: uma revisão sistemática. Revista Eletrônica Acervo Saúde, 2020; 44: e3325.

22. SRIDHAR VS, et al. Argumentando a favor do uso combinado de inibidores de SGLT2 e agonistas do receptor GLP1 para proteção cardiorrenal. Brazilian Journal of Nephrology, 2020; 42(4): 467-477.

23. TENTOLOURIS A, et al. SGLT2 Inhibitors: A Review of Their Antidiabetic and Cardioprotective Effects. International journal of environmental research and public health, 2019; 16(16): 2965.

24. TOYAMA T, et al. Effect of SGLT2 inhibitors on cardiovascular, renal and safety outcomes in patients with type 2 diabetes mellitus and chronic kidney disease: a systematic review and meta-analysis. Diabetes, Obesity and
Metabolism, 2019;21(5): 1237-1250.

25. USMAN MS, et al. Sodium-glucose co-transporter 2 inhibitors and cardiovascular outcomes: A systematic review and meta-analysis, 2018. European Journal of Preventive Cardiology, 2018; 25(5): 495-502.

26. UTHMAN L, et al. Class effects of SGLT2 inhibitors in mouse cardiomyocytes and hearts: inhibition of $\mathrm{Na}+/ \mathrm{H}+$ exchanger, lowering of cytosolic Na+ and vasodilation. Diabetologia, 2018; 61(3): 722-726.

27. VERMA S, MCMURRAY JJV. SGLT2 inhibitors and mechanisms of cardiovascular benefit: a state-of-the-art review. Diabetologia, 2018; 61(10): 2108-2117.

28. ZELNIKER TA, et al. SGLT2 inhibitors for primary and secondary prevention of cardiovascular and renal outcomes in type 2 diabetes: a systematic review and meta-analysis of cardiovascular outcome trials. The Lancet, 2019; 392(10166): 31-39.

29. ZINMAN B, et al. Empagliflozin, Cardiovascular Outcomes, and Mortality in Type 2 Diabetes. N Engl J Med. 2015; 373(22): 2117-28. 